\title{
Etnoconhecimento e ofidismo na comunidade Rolim de Moura do Guaporé, Alta Floresta do Oeste, Sudoeste da Amazônia brasileira
}

\begin{abstract}
A relação entre sociedade e natureza e todos os componentes nela existentes é primitiva, assim como a utilização que o mesmo faz dos recursos naturais disponíveis. Nesse contexto, é importante conhecer a cultura dos povos sobre os grupos animais, para o auxiliar em medidas de educação ambiental e conservação das espécies. O objetivo deste trabalho consistiu em verificar o conhecimento empírico e os casos de ofidismo ocorrentes no distrito de Rolim de Moura do Guaporé, município de Alta Floresta do Oeste, RO, bem como analisar a relação desta comunidade para com as serpentes. Foram aplicados 29 questionários em pessoas moradoras do distrito, de ambos os sexos, com idade superior a 18 anos e que permitiram a aplicação da pesquisa. A taxa dos encontros dos moradores com serpentes foi considerada 'muito frequente', com cerca de $96 \%$ das pessoas afirmando avistar estes animais pelo menos uma vez na semana. Além disso, citam Bothrops atrox e Boa constrictor como as serpentes mais frequentes no entorno da comunidade. Em caso de encontro com serpentes, $72 \%$ dos entrevistados disseram não matar o animal caso este não seja potencialmente peçonhento. No entanto, os modos de identificação usados pela população são bastante limitados e baseados em aspectos morfológicos que não são válidos, como formato da cabeça e da cauda. Os resultados demonstraram que os moradores mantêm uma relação de respeito com as serpentes, entendendo que apesar dos desafios dessa convivência, elas apresentam um importante papel chave no equilíbrio ecológico e que sua preservação beneficia toda a comunidade.
\end{abstract}

Palavras-chave: Etnoherpetologia; Sociedade/natureza; Serpentes; Acidentes ofídicos.

\section{Ethnocognition and snakebite in the Rolim de Moura do Guaporé community, Alta Floresta do Oeste, Southwestern Brazilian Amazon}

\begin{abstract}
The relationship between man and nature and all its components are primitive, as well as its use of available natural resources. In this context, it is important to know the culture of different peoples about animal groups, assisting in measures of environmental education and conservation of species. The objective of this research was to verify the empirical knowledge and cases of snakebites that occurred in the district of Rolim de Moura do Guaporé, municipality of Alta Floresta do Oeste, RO, as well as analyzing the relationship of this community with the snakes. To obtain data, a semi-structured questionnaire was used in order to achieve the objectives defined in the research. The questionnaires were issued to 29 people living in the district, of both sexes, over the age of 18 and who allowed the application of the survey. The rate of residents' encounters with snakes was considered "very frequent", with around $96 \%$ of people claiming to see these animals at least once a week. In addition, they cite Bothrops atrox and Boa constrictor as the most frequent snakes around the community. In the event of encounters with snakes, $72 \%$ of respondents said that they would not kill the animal as long as it were not potentially venomous. However, the modes of identification used by the population are well known and based on morphological aspects that are not valid, such as the shape of the head and tail. The results showed that the residents maintain a respectful relationship with the snakes, understanding that despite the controversies of this coexistence, they play an important role in ecological balance and that their preservation benefits the entire community.
\end{abstract}

Keywords: Ethnoherpetology; Society/nature; Snakes; Snakebite accidents.

Topic: Conservação da Biodiversidade

Reviewed anonymously in the process of blind peer.
Received: 03/02/2021

Approved: 25/02/2021
Katiele de Jesus Sacoman (iD)

Universidade Federal de Rondônia, Brasil

http://lattes.cnpq.br/4349410154070080

http://orcid.org/0000-0003-3944-3397

katiele.sacoman@hotmail.com

Larissa Gabriela Araujo Goebel (ic

Universidade do Estado de Mato Grosso, Brasil

http://lattes.cnpq.br/6245811015684023

http://orcid.org/0000-0002-1320-21843

larissagabriela goebel@hotmail.com

Eder Correa Fermiano (iD)

Universidade do Estado de Mato Grosso, Brasil

http://lattes.cnpq.br/5020850029576982

http://orcid.org/0000-0002-1465-0675982

edderfermiano@gmail.com

\author{
Mariza de Lima Schiavi (iD) \\ Faculdade de Ciências Biomédicas de Cacoal, Brasil \\ http://lattes.cnpq.br/2359971740519312 \\ http://orcid.org/0000-0003-2952-6033 \\ mariza_schiavi@outlook.com \\ Victor Hugo de Oliveira Henrique (iD \\ Universidade do Estado de Mato Grosso, Brasil \\ http://lattes.cnpq.br/3439022161736672 \\ http://orcid.org/0000-0002-7019-4088 \\ hugo31 oh@hotmail.com \\ Rodrigo Lemes da Silva (iD) \\ Faculdade de Ciências Biomédicas de Cacoal, Brasil \\ http://lattes.cnpq.br/1276225085722861 \\ http://orcid.org/0000-0001-9929-6291 \\ rlscacoal@hotmail.com
}

Referencing this:

SACOMAN, K. J.; GOEBEL, L. G. A.; FERMIANO, E. C.; SCHIAVI, M. L.; HENRIQUE, V. H. O.; SILVA, R. L.. Etnoconhecimento e ofidismo na comunidade Rolim de Moura do Guaporé, Alta Floresta do Oeste, Sudoeste da Amazônia brasileira. Revista Ibero Americana de Ciências Ambientais, v.12, n.2, p.177-185, 2021. DOI:

http://doi.org/10.6008/CBPC2179-6858.2021.002.0018 


\section{INTRODUÇÃO}

As serpentes formam um dos grupos mais diversificados no mundo, sendo que no Brasil são registradas 412 espécies (NOGUEIRA et al., 2019). Muitas destas espécies apresentam grande importância médica (Famílias Elapidae e Viperidae) e desempenham papel importante no equilíbrio dos ecossistemas, regulando a densidade de animais que consomem, além de servir de alimento para outras espécies (LIMA et al., 2018). Este grupo está presente nos mais variados tipos de habitats, desde florestas úmidas a regiões semiáridas, apresentando uma diversidade de características morfológicas e ecológicas importantes para a identificação e conhecimento da ecologia de cada espécie (MELGAREJO, 2009).

É notável que as serpentes possuem uma estreita relação com a sociedade desde os tempos primitivos, sendo citadas em diversas culturas como parte de lendas e mitos que atravessam gerações (VIZOTTO, 2003; BERNARDE, 2012). Apesar de muitas vezes serem reverenciadas devido a essas crendices, algumas lendas também proporcionam o extermínio das serpentes independentemente de serem peçonhentas ou de apresentarem perigo. Tais ações são impulsionadas muitas vezes pelo medo e falta de conhecimento (FREITAS, 2003; BAPTISTA et al., 2008).

Nesse contexto, a etnociência torna-se uma importante ferramenta para conhecer a compreensão e percepção que diversos povos têm do mundo ao seu redor (BEGOSSI, 1993). Estudos como de Vasconcelos Neto et al. (2018) relacionados à cultura de populações tradicionais demonstram que cada comunidade tem sua própria maneira de perceber o mundo e de fazer uso dos recursos nele disponíveis, contribuindo para descobertas de novas espécies e/ou mesmo conservação daquelas já descritas.

Deste modo, através de ações educativas relacionadas ao conhecimento da população, é possível promover a conservação das espécies locais e também tornar a relação da sociedade com a natureza mais harmoniosa (LIMA et al., 2018). Sendo assim, o objetivo deste trabalho consistiu em verificar o conhecimento empírico e os casos de ofidismo ocorrentes em uma comunidade tradicional no sudoeste da Amazônia Brasileira, bem como analisar a relação da comunidade com o grupo de serpentes.

\section{MATERIAIS E MÉTODOS}

\section{Área de Estudo}

O estudo foi realizado no distrito de Rolim de Moura do Guaporé (13ํํ'58.4"S 62¹6'33.7"W) (Figura 1), pertencente ao município de Alta Floresta d'Oeste - RO, no sudoeste da Amazônia brasileira. A comunidade é composta por etnias indígenas, remanescentes de quilombolas, extrativistas, estrangeiros de origem boliviana, fazendeiros e outros que apresentam interesses diferenciados sobre o local. $\mathrm{O}$ distrito encontra-se ilhado pelas águas dos rios Mequéns, Colorado e Guaporé, impedindo o acesso à comunidade por via terrestre (GOEBEL et al., 2019). De acordo com a classificação de Köppen's, o clima da região é do tipo Am, com precipitação média anual entre 1900 e 2200 mm e temperatura média anual variando entre 24 e 26 ㄷ (ALVARES et al., 2013). 

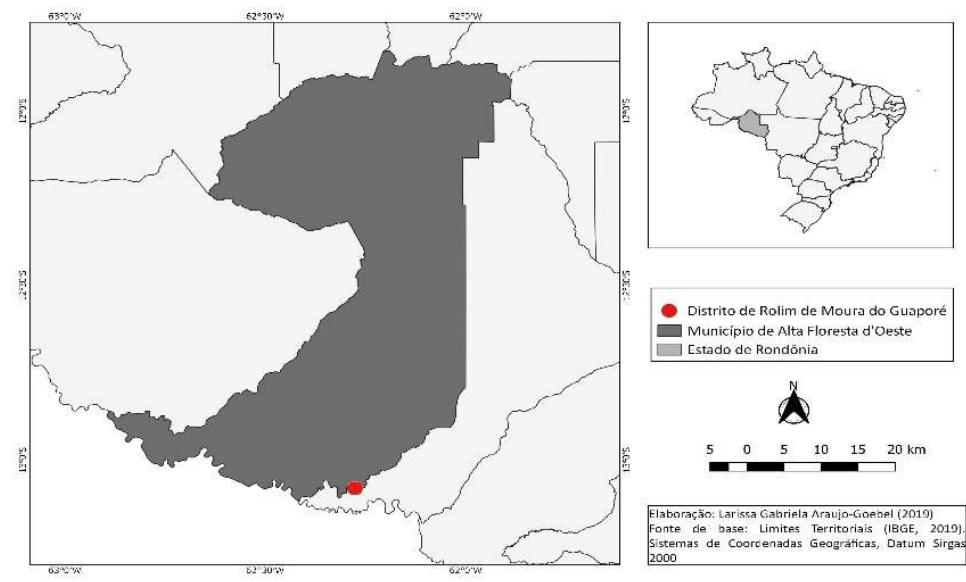

Figura 1: Localização do distrito de Rolim de Moura do Guaporé, Alta Floresta - RO.

\section{Coleta de dados}

Para obtenção dos dados foi elaborado um questionário semiestruturado (Quadro 1). O mesmo foi aplicado a moradores do distrito, de ambos os sexos, com idade igual ou superior a 18 anos e que aceitaram participar da pesquisa. Moradores foram abordados de forma aleatória e convidados a responder ao questionário. Para todos os participantes foi detalhado os objetivos da pesquisa de modo que todos estivessem cientes da finalidade do estudo. Pessoas que optaram por não participar da pesquisa ou não estavam presentes na residência no momento da visita não foram incluídos na amostra.

Quadro 1: Questionário sobre a temática a ser pesquisada.

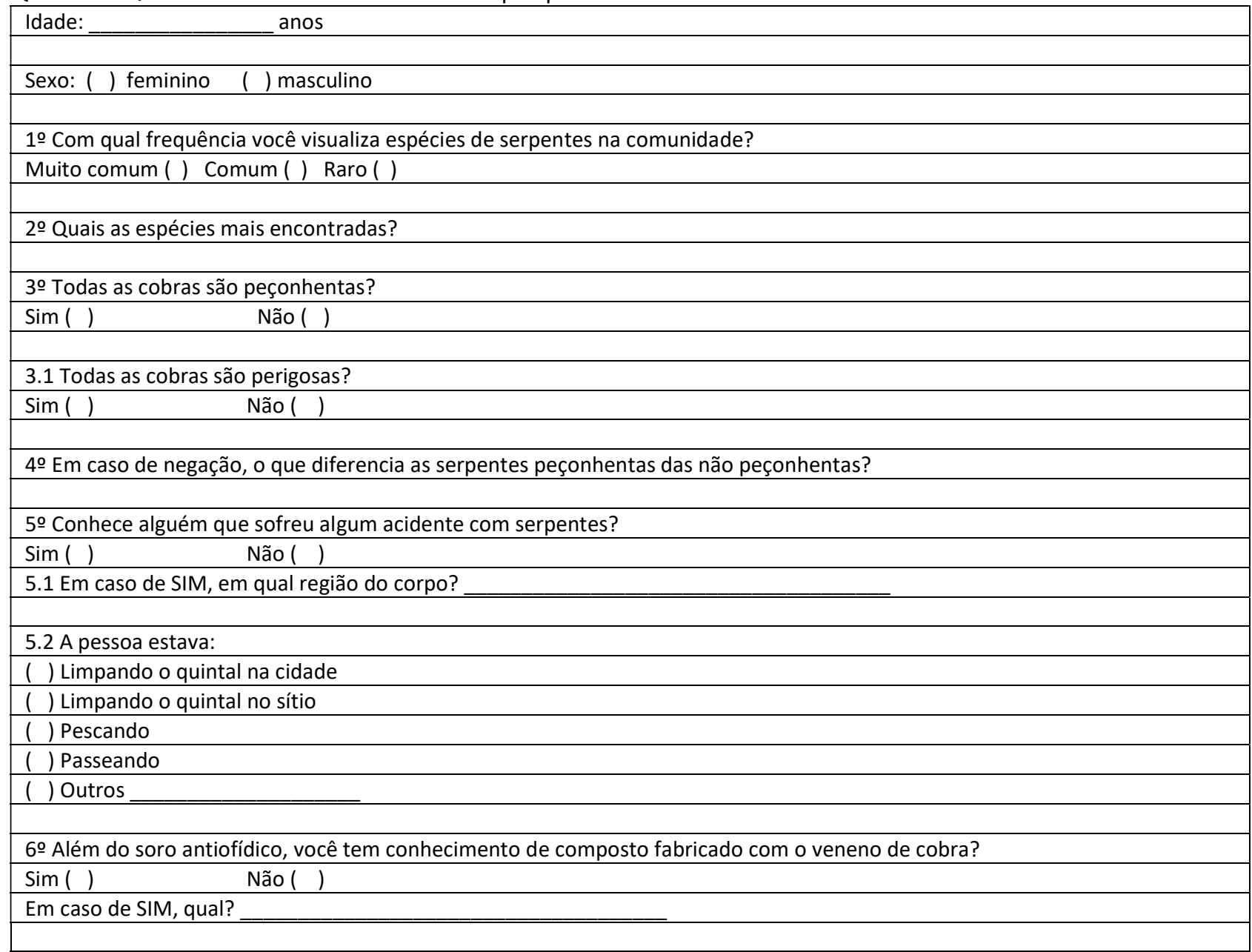




\begin{tabular}{|l|}
\hline 7 Se você encontrar uma cobra em um ambiente campestre (campo, mato) o que você faria? \\
\hline ( ) mataria \\
\hline ( ) sairia correndo \\
\hline ( ) deixaria ela passar \\
\hline 8o Em sua opinião, a cobra tem alguma serventia para o meio ambiente? \\
\hline Sim ( ) Não ( ) \\
\hline Em caso de SIM, qual? \\
\hline 9o Já ouviu alguma crença envolvendo serpentes? \\
\hline Sim ( ) Não ( ) \\
\hline
\end{tabular}

O questionário foi aplicado nos dias 15 e 16 de novembro de 2018. Entre os tópicos abordados, buscamos verificar a frequência de encontros com serpentes pelos moradores nos arredores da comunidade. Os encontros foram classificados em: "muito comum", quando estes eram feitos pelo menos uma vez por semana; "comum", quando ocorriam pelo menos uma vez ao mês; e "raro" quando as serpentes eram visualizadas com intervalos superiores a um mês. Para identificação científica das espécies descritas pelos moradores, foram apresentadas figuras das espécies presentes em guias de identificação (MARQUES et al., 2005; BERNARDE et al., 2012; BERNARDE, 2012). A nomenclatura científica adotada neste estudo segue Nogueira et al. (2019), entre outros autores, e os nomes populares usados neste trabalho representam o que usualmente é usado na comunidade. A análise dos dados foi realizada por frequência simples.

\section{RESULTADOS}

Ao todo 29 pessoas do distrito foram entrevistadas, destas, 28 pessoas $(96,56 \%)$ disseram ser 'muito comum' a visualização de serpentes na comunidade, enquanto apenas uma pessoa afirmou não ter visto nenhuma serpente a vários meses, sendo considerado 'raro'. Tendo em vista a alta taxa de encontro com serpentes, os moradores discorreram sobre as espécies mais observadas no local, sendo destacada a jararaca (Bothrops atrox) e a jiboia (Boa constrictor). Um, dentre os 29 moradores abordados, não soube identificar uma das espécies visualizadas citando apenas o nome popular (Figura 1).

A respeito de considerarem ou 'não', todas as serpentes peçonhentas, o número de respostas negativas foi amplamente maior que o número de positivas. Sendo assim, $82,76 \%$ das pessoas afirmaram que nem todas as serpentes possuem peçonha, $17,24 \%$ afirmaram que mesmo que pouco, todas eram peçonhentas. Quando perguntado se todas as serpentes são perigosas, 58,62\% disseram que não e 41,68\% afirmaram que sim, mostrando um maior equilíbrio entre as opiniões.

Com relação às características que são consideradas mais importantes para a diferenciação das serpentes como peçonhentas e não peçonhentas, alguns fatores foram citados (Figura 2). Neste quesito, os entrevistados citaram mais de uma característica, por este motivo, o total de opiniões é maior que o número total de entrevistados. Dentre as respostas, duas pessoas disseram que 'depende da espécie', porém, não deram maiores esclarecimentos sobre suas opiniões.

Os casos de ofidismo na localidade também foram investigados, sendo que 22 entrevistados $(75,86 \%)$ relataram conhecer algum morador acidentado. Além disso, foi relatado que os acidentes ocorrem com maior frequência no período chuvoso. Entre os relatos dos moradores, 100\% dos acidentes ocorreram 
enquanto as pessoas limpavam seus quintais, em área considerada urbana da comunidade. Entre as partes do corpo com maior número de acidentes ofídicos se destaca os membros inferiores com 93\% dos casos de ofidismo, enquanto a incidência de casos nos membros superiores é de 6,90\%.

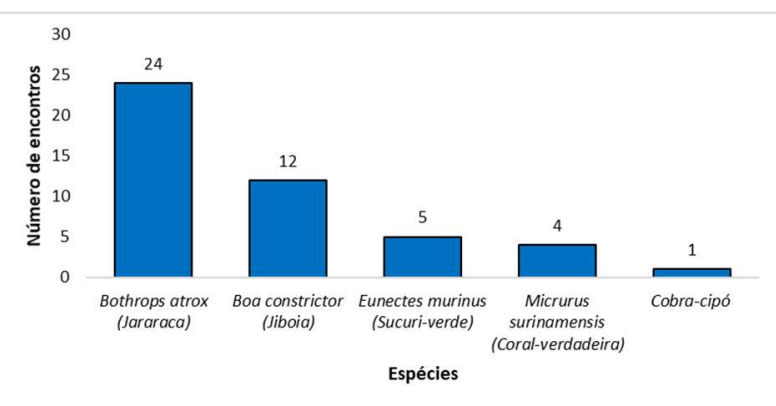

Figura 1: Espécies de serpentes visualizadas por moradores na comunidade de Rolim de Moura do Guaporé, Alta Floresta - RO.

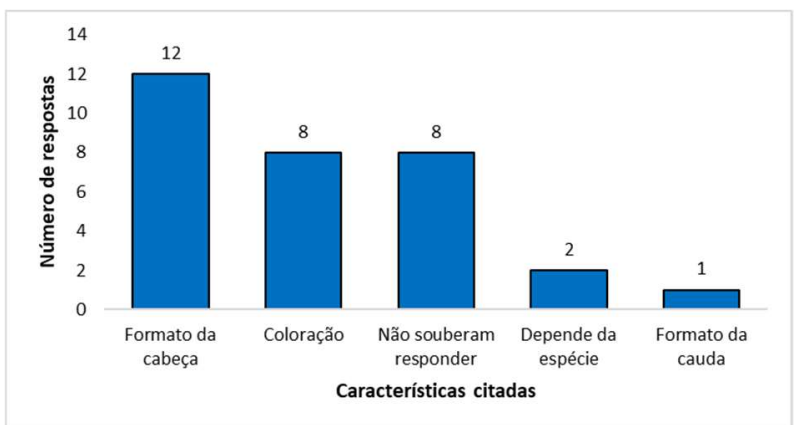

Figura 2: Características apontadas pelos moradores como forma de distinção entre serpentes peçonhentas e não peçonhentas em Rolim de Moura do Guaporé, Alta Floresta - RO.

Quando questionados sobre a importância das serpentes, embora timidamente, os moradores destacaram desde o controle de pragas quanto na construção e manutenção do equilíbrio ecológico. Apesar disso alguns moradores disseram não haver importância alguma na existência das serpentes e outros não souberam responder (Figura 3).

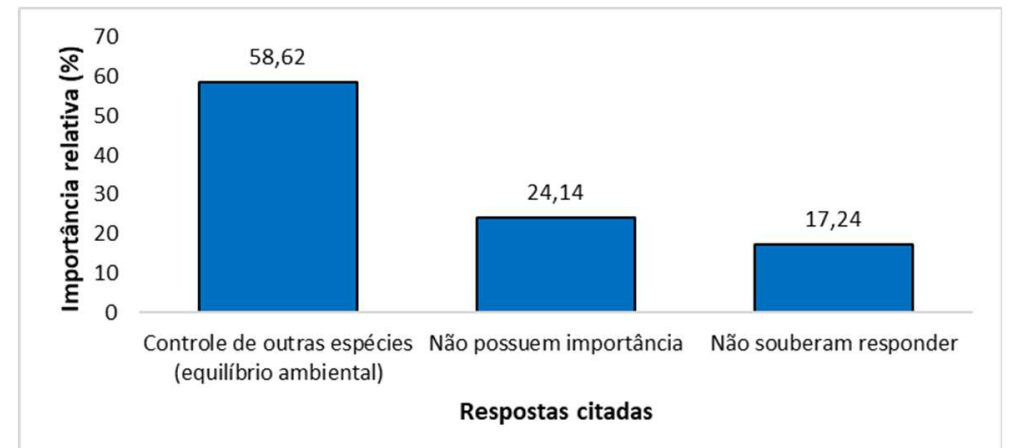

Figura 3: Importância das serpentes na visão dos moradores entrevistados em Rolim de Moura do Guaporé, Alta Floresta - RO.

\section{DISCUSSÃO}

Botrhops atrox (jararaca) e Boa constrictor (jiboia) ocuparam posições de destaque entre as serpentes mais encontradas pelos moradores. Isto pode ser explicado pelo fato de serem animais de hábitos alimentares generalistas, tendo em suas dietas principalmente pequenos mamíferos, aves, anfíbios e lagartos, que são encontrados em abundância próximo às habitações humanas (BERNARDE et al., 2010; FERMIANO et al., 2018; SANCHES et al., 2018). Além disso, B. constrictor apresenta grande porte, fato que a impede de passar despercebida aos olhos humanos e possibilite um maior número de registros (FERMIANO et al., 2018).

Outras duas espécies citadas pelos entrevistados foram: Eunectes murinus (sucuri-verde), sendo também uma serpente de grande porte e muito temida principalmente por existir diversas lendas sobre este animal (BERNARDE, 2012); e Micrurus surinamensis uma das cobras corais-verdadeiras mais encontradas na 
Amazônia e que, apesar de ser pouco agressiva, possui um veneno muito potente com propriedades neurotóxicas (FREITAS, 2003). O encontro destas espécies no local pode ser explicado pelo fato da comunidade estar localizada em uma ilha cercada por rios e estas serpentes possuírem hábitos aquáticos (MARTINS et al., 1998; BERNARDE et al., 2010; BERNARDE, 2012).

Dentre as características citadas pelos moradores, para diferenciar as serpentes peçonhentas das não peçonhentas, nenhuma delas é cientificamente eficaz. Um exemplo é o formato triangular da cabeça, presente em viperídeos, comumente citado pelas pessoas e inclusive já observado em livros didáticos, como sendo uma característica importante para esta diferenciação (SANTOS et al., 1995). Porém, esta é uma característica comum a várias serpentes não peçonhentas, como é o caso da jiboia, citada por muitos moradores como uma das mais abundantes da região. Outras espécies não peçonhentas, podem triangular a cabeça, aparentemente, na intenção de imitar os viperídeos e intimidar seus predadores (BERNARDE, 2012).

A coloração foi citada pelos moradores, principalmente relacionadas às cobras corais-verdadeiras que em sua maioria, utilizam cores fortes e vibrantes como forma de aviso de perigo (BERNARDE, 2012). No entanto muitas são as serpentes não peçonhentas que as mimetizam, e também as corais-verdadeiras que não possuem cores aposemáticas, ou padrão coralino tradicional (vermelho, preto e branco ou amarelo) (GRANTSAU, 2013). Por exemplo, Micrurus albicinctus encontrada no estado de Rondônia (NOGUEIRA et al. 2019) possui coloração preta com anéis finos e pontilhados de branco (BERNARDE, 2012), e a recém-descrita M. boicora com a região dorsal negra e apenas pequenas porções do ventre com manchas avermelhadas (BERNARDE et al., 2018).

O formato da cauda foi mencionado por apenas um entrevistado, sendo este também um método não confiável para a diferenciação entre serpentes peçonhentas das não peçonhentas (SANTOS et al., 1995; GRANTSAU, 2013). Apesar disso, pode ser utilizado para diferenciar os viperídeos: jararaca, com a cauda lisa; cascavel, com um chocalho na ponta da cauda; e a bico-de-jaca, com as escamas da cauda eriçadas e a última escama em forma de espinho (SANTOS et al., 1995; GRANTSAU, 2013).

No Brasil, as principais características para determinação de serpentes peçonhentas e não peçonhentas são a fosseta loreal e a dentição do tipo solenóglifa para espécies da família Viperidae (jararacas, cascavel e bico-de-jaca) e do tipo proteróglifa para a família Elapidae (cobras corais-verdadeiras) (SANTOS et al., 1995; MARQUES et al., 2005; BERNARDE, 2012; GRANTSAU, 2013). Neste sentido, percebese um método arcaico e pouco eficiente para a diferenciação das espécies por parte da comunidade, evidenciando a necessidade de ações educativas.

De acordo com Turci et al. (2009) o pico das atividades de algumas serpentes, em específico B. atrox ocorre no período chuvoso, o que ocasiona um alto índice de acidentes com esta espécie. Como mencionado pelos entrevistados, os acidentes na região acometem principalmente os membros inferiores, reforçando a importância do uso de botas ou botinas com cano alto (BERNARDE, 2012). Em função do grande número de acidentes no local, os moradores foram instigados a relatar se possuíam conhecimento de compostos que pudessem auxiliar na neutralização dos sintomas em caso de acidentes. Cerca de $80 \%$ dos entrevistados 
responderam que não conhecem qualquer composto, e os demais $20 \%$ relataram conhecer o composto "Específico Pessoa", como um dos mais utilizados na região em casos de ofidismo. Tal composto é um fitoterápico utilizado em muitas comunidades tradicionais como modo de profilaxia e auxílio de picadas de animais peçonhentos com ênfase das serpentes, contudo, não há comprovação científica da eficácia do mesmo (BORGES et al., 1996; BERNARDE et al. 2017).

Mesmo que a relação entre sociedade e serpentes tenha sido potencializada durante as últimas décadas, principalmente devido à invasão do habitat natural pelo ser humano, ainda pouco se conhece da ecologia desses répteis, o que acarreta em extermínio descontrolado de diversas espécies, sendo elas peçonhentas ou não (MOURA et al., 2010). Quando questionados sobre a forma de agir caso encontrassem uma serpente, independente de possuírem peçonha ou não, apesar da demonstração de medo por grande parte dos moradores, cerca de $72 \%$ disseram que, caso não apresentasse risco para si nem para terceiros, a 'deixaria passar' e ir embora. Os demais afirmaram que matariam o animal.

Assim como em outras localidades, lendas e crendices fazem parte do cotidiano da comunidade de Rolim de Moura do Guaporé. Entre as principais lendas citadas estão: cobras que andam somente em casal; cobras que hipnotizam; cobras mal matadas que se vingam e cobras que bebem leite. Todas as lendas citadas foram enfatizadas com relatos sobre experiências que pessoas próximas ao morador teriam vivido. Neste sentido, é de extrema importância que se conheça o modo com que as pessoas veem esses animais, levando em conta que isso está intimamente ligado com as conservações dos mesmos (BERNARDE, 2012).

As serpentes desempenham papel fundamental no equilíbrio ambiental (MARQUES et al., 2005), e algumas apresentam, através de seu veneno, potencial para a produção de fármacos e produtos para fins cirúrgicos (MARTINS et al., 2008; BERNARDE et al., 2017). Contudo, seus benefícios ainda são pouco conhecidos em muitas localidades. Sendo assim, este estudo demonstra que ainda há muito o que se fazer, principalmente em relação a medidas de educação ambiental, visto que muitos mitos ainda são disseminados sobre as serpentes e muitos animais são mortos independentemente de serem potencialmente peçonhentos. Recomenda-se ações educacionais no âmbito escolar a fim de iniciar processos de sensibilização para crianças e jovens, como meio de demonstrar a real importância das serpentes, bem como transmitir informações de prevenção de acidentes e primeiros socorros.

\section{CONCLUSÕES}

A comunidade estudada demonstrou que possui uma relação de respeito e harmonia com as serpentes, e que mesmo com todas as crendices, misticismos e acidentes ofídicos em alto índice em algumas épocas do ano, os moradores compreendem que as serpentes possuem um papel auxiliador no equilíbrio ambiental, não só da localidade como de outros habitats e que por isso evitam matar quando as mesmas não apresentam perigo para si nem para os demais.

Os moradores deixaram claro que a educação ambiental sobre a importância das serpentes e aprimoramento de seus conhecimentos são bem-vindos, uma vez que quanto mais se conhece a respeito do ambiente em que vivem e sobre os organismos com os quais compartilham o espaço, é possível trazer 
segurança para a comunidade e contribuir com uma relação harmoniosa para ambos.

AGRADECIMENTOS: a Coordenação de Aperfeiçoamento de Pessoal de Nível Superior (CAPES) pelas bolsas de mestrado concedida aos autores.

\section{REFERÊNCIAS}

ALVARES, C. A.; STAPE, J. L.; SENTELHAS, P. C.; GONÇALVES, J. L. M.; SPAROVEK, G.. Köppen's climate classification map for Brazil. Meteorologische Zeitschrift, v.22, n.6, p.711-728, 2013. DOI: http://doi.org/10.1127/0941-2948/2013/0507

BAPTISTA, G. C. S.; COSTA NETO, E. M.; VALVERDE, M. C. C.. Diálogo entre concepções prévias dos estudantes e conhecimento científico escolar: relações sobre os Amphisbaenia. Revista Ibero-Americana de Estudos em Educação, v.47, n.2, p.1-16, 2008. DOI: https://doi.org/10.35362/rie4722370

BEGOSSI, A.. Ecologia humana: um enfoque das relações homem-ambiente. Interciencia, v.18, n.3, p.121-132, 1993.

BERNARDE, P. S.; TURCI, L. C. B.; MACHADO, R. A.. Serpentes do Alto Juruá, Acre: Amazônia brasileira. Rio Branco: Edufac, 2017.

BERNARDE, P. S.. Anfíbios e répteis: introdução ao estudo da herpetofauna brasileira. Curitiba: Anolis Books, 2012.

BERNARDE, P. S.; ABE, A. S.. Hábitos alimentares de serpentes em Espigão do Oeste, Rondônia, Brasil. Biota Neotropica, v.10, n.1, p.167-173, 2010. DOI: https://doi.org/10.1590/S1676-06032010000100017

BERNARDE, P. S.; TURCI, L. C. B.; ABEGG, A. D.; FRANCO, F. L.. A remarkable new species of coralsnake of the Micrurus hemprichii species group from the Brazilian Amazon. Salamandra, v.54, n.4, p.249-258, 2018.

BERNARDE, P. S.; ALBUQUERQUE, S.; BARROS, T. O.; TURCI, L. C. B.. Serpentes do Estado de Rondônia, Brasil. Biota Neotropica, Campinas, v.12, n.3, p.154-182, 2012. DOI: https://doi.org/10.1590/S1676-06032012000300018

BORGES, C. C.; CAVALCANTI NETO, A. J.; BOECHAT, A. L.; FRANCISCO, C. H.; ARRUDA, L. F. M. R.; SANTOS, M. C.. Eficácia da espécie vegetal Peltodon radicans POHL (Labiatae = Lamiaceae) na neutralização da atividade edematogênica e a ineficácia do extrato vegetal Específico Pessoa na neutralização das principais atividades do veneno de Bothrops atrox. Revista da Universidade do Amazonas, v.1, p.97-113, 1996.

GRANTSAU, R.. As serpentes peçonhentas do Brasil. São Carlos: Vento Verde, 2013.

FERMIANO, E. C.; ROBERTO, W. S.; SOUZA, J.; SILVA, P. J. G.; SURUI, F. G.; SILVA, A. P.. Serpentes registradas ocasionalmente no município de Alvorada do Oeste-RO, entre 2016 e 2017. Revista Saber Científico, v.7, n.1, p.9198, 2018.

FREITAS, M. A.. Serpentes brasileiras. Lauro de Freitas: Malha-de-Sapo-Publicações, 2003.
GOEBEL, L. G. A.; SACOMAN, K. J.; SCHIAVI, M. L.; PEREIRA, L. S.; SILVEIRA, I. P.; GONÇALVES, M. S.. Etnoconhecimento da mastofauna do distrito de Rolim de Moura do Guaporéo RO, Amazônia, Brasil. Revista Brasileira de Ciências da Amazônia, v.8, n.3, p.001-008, 2019.

LIMA, B. S. L.; SOUZA, M. M.; SOUTO, N. L.; BARROS, A. B. Investigando o conhecimento etnoherpetológico dos cafeicultores sobre as serpentes do município de inconfidentes, Minas Gerais. Ethnoscientia, v.2, p.1-13, 2018.

MARQUES, O. A. V.; ETEROVIC, A.; STRUSSMANN, C.; SAZIMA, I.. Serpentes do Pantanal: Guia ilustrado. Ribeirão Preto: Holos, 2005.

MARTINS, M.; OLIVEIRA, M. E.. Natural history of snakes in forests of the Manaus region, Central Amazonia, Brazil. Herpetological Natural History, v.6, n.2, p.78-150, 1998.

MARTINS, M.; MOLINA, F. B.. Panorama geral dos répteis ameaçados do Brasil. In: MACHADO, A. B. M.; DRUMMOND, G. M.; PAGLIA, A. P.. Livro vermelho da fauna brasileira ameaçada de extinção. Brasília: Ministério do Meio Ambiente, 2008. p.327-73.

MELGAREJO, A. R.. Serpentes Peçonhentas do Brasil. In: CARDOSO, J. L.; FRANÇA, F. O. S.; WEN, F. H.; MÁLAQUE, C. M. S.; HADDAD JUNIOR, V.. Animais peçonhentos no Brasil: biologia, clínica e terapêutica. 2 ed. São Paulo: SARVIER, 2009.

MOURA, M. R.; COSTA, H. C.; SÃO-PEDRO, V. A.; FERNANDES, V. D.; FEIO, R. N.. The relationship between people and snakes in eastern Minas Gerais, southeastern Brazil. Biota Neotropica, v.10, n.4, 2010. DOI: https://doi.org/10.1590/S1676-06032010000400018

NOGUEIRA, C. C.; ARGÊLO, A. J. S.; ARZAMENDIA, V.; AZEVEDO, J. A.; BARBO, F. E.; BÉRNILS, R. S.; BOLOCHIO, B. E.; BORGES-MARTINS, M.; BRASIL-GODINHO, M.; BRAZ, H.; BUONONATO, M. A.; CISNEROS-HEREDIA, D. F.; COLLI, G. R.; COSTA, H. C.; FRANCISCO, L.; FRANCO, F. L.; GIRAUDO, A.; GONZALEZ, R. C.; GUEDES, T. B.; HOOGMOED, M. S.; MARQUES, O. A. V.; MONTINGELLI, G. G.; PASSOS, P. PRUDENTE, A. L. C.; RIVAS, G. A.; SANCHEZ, P. M.; SERRANO F. C.; SILVA, J. R. N. J.; STRÜSSMANN, C.; VIEIRA-ALENCAR, J. P. S.; ZAHER, H.; SAWAYA, R. J.; MARTINS, M.. Atlas of Brazilian snakes: verified point-locality maps to mitigate the Wallacean shortfall in a megadiverse snake fauna. South American Journal of Herpetology, v.14, n.1, p.1-274, 2019.

SANCHES, P. R.; SANTOS, F. P.; GAMA, C. S.; COSTA-CAMPOS, C. E.. Predation on Iguana iguana (Squamata: Iguanidae) by Boa constrictor (Squamata: Boidae) in a fluvial island in the Amazonas river, Brazil, including a list of saurophagy events 
with Boa constrictor as predator. Cuadernos de

Herpetología, v.32, n.2, p.129-132, 2018. DOI:

http://doi.org/10.31017/CdH.2018.(2018-002)

SANTOS, M. C.; MARTINS, M.; BOECHAT, A. L.; NETO, R. P. S.; OLIVEIRA, M. E.. Serpentes de interesse médico da

Amazônia. Manaus: Universidade do Amazonas, 1995.

SILVA JUNIOR, N. J.. The snakes from Samuel hydroeletric power plant and vicinity, Rondônia, Brasil. Herpetological Natural History, v.1, p.37-86, 1993.

TURCI, L. C. B.; ALBUQUERQUE, S.; BERNARDE, P. S.; MIRANDA, D. B.. Uso do hábitat, atividade e comportamento de Bothriopsis bilineatus e de Bothrops atrox (Serpentes: Viperidae) na floresta do Rio Moa, Acre, Brasil. Biota Neotropica, v.9, n.3, 2009. DOI:

https://doi.org/10.1590/S1676-06032009000300020

VASCONCELOS NETO, L. B.; SILVA, A. S. G.; BRITO, I. A. S.; CHALKIDIS, H. M.. O conhecimento Tradicional Sobre as Serpentes em uma Comunidade Ribeirinha no Centro-Leste da Amazônia. Ethnoscientia, v.3, p.1-7, 2018. DOI: http://doi.org/10.22276/ethnoscientia.v3i0.157

VIZOTTO, L. D.. Serpentes: lendas, mitos, superstições e crendices. São Paulo: Plêiade, 2003.

A CBPC - Companhia Brasileira de Produção Científica (CNPJ: 11.221.422/0001-03) detém os direitos materiais desta publicação. Os direitos referem-se à publicação do trabalho em qualquer parte do mundo, incluindo os direitos às renovações, expansões e disseminações da contribuição, bem como outros direitos subsidiários. Todos os trabalhos publicados eletronicamente poderão posteriormente ser publicados em coletâneas impressas sob coordenação da Sustenere Publishing, da Companhia Brasileira de Produção Científica e seus parceiros autorizados. Os (as) autores (as) preservam os direitos autorais, mas não têm permissão para a publicação da contribuição em outro meio, impresso ou digital, em português ou em tradução. 